\title{
Muzlifah Haniffa-a new era for collaborative and supportive medical research
}

\author{
Muzlifah Haniffa is a dermatologist working at Newcastle University and Wellcome Sanger Institute. Her \\ achievements were recognized by the 2019 Foulkes Foundation Medal awarded by the Academy of Medical \\ Sciences. She also plays a pioneering role in the Human Cell Atlas initiative.
}

\section{Muzlifah Haniffa}

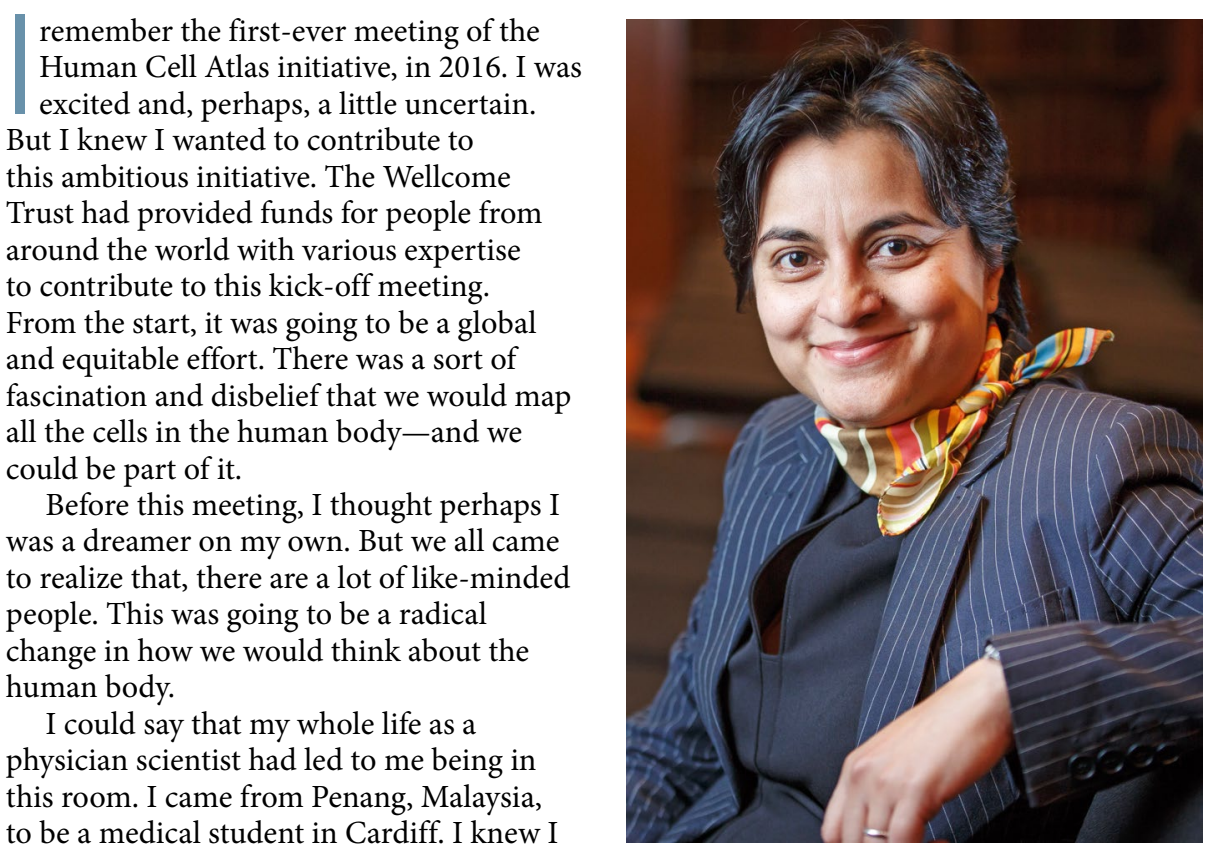
to be a medical student in Cardiff. I knew I wanted to do a substantive piece of research toward a PhD. There are several points at which a clinician can go out of clinical training to do research in the UK; I did this during my specialty training so I could align my research and clinical fields. I secured a research training fellowship from the charity Action Medical Research while undertaking dermatology training in Newcastle, studying antigen-presenting cells in the skin. I want to emphasize the importance of fellowships for my clinical research, to weather the highs and lows of academia. Clinicians have to be freed up from their clinical workload to do their research.

I worked under the joint supervision of the hematology, rheumatology and dermatology departments. This was a stroke of luck! I learned that understanding how people think and approach problems from another field drives innovation of thinking in your own field.

After I completed my dermatology training, a Wellcome Clinical Intermediate Fellowship on the origin and function of
Credit: BigT Images, Academy of Medical Sciences

antigen-presenting cells in the skin took me to Singapore for a year; this culminated in a cross-species homology framework, based on transcriptome analysis, for antigenpresenting cells in human and mouse.

I became involved in the creation of an atlas of human blood dendritic cells and monocytes with researchers at the Broad Institute of MIT and Harvard. There I realized the power of such a newly emerging field of research: single cell genomics. I also learned about the importance of true collaboration. You could not achieve this project with the capabilities of genomics, computational expertise, or a biomedical approach alone.

Historically, medicine has gone down the route of specialization. But give a problem to people who have been taught the same way and they will approach it in a singular fashion. For better innovation in research, you need collaboration, equality and diversity. The Human Cell Atlas initiative (https://www.humancellatlas.org) provided the precise platform for multidisciplinary team science to map the 37 trillion cells in the human body by singlecell genomics. A new culture of researchers working together around the world in a complementary fashion and sharing their findings rapidly and openly quickly emerged.

The Human Cell Atlas initiative provided me with the opportunity to decode the human immune system during development, an area that had been a 'black box' hitherto. This research is in partnership with groups at the Wellcome Sanger Institute (the Teichmann, Behjati and Clatworthy groups and others), the Human Developmental Biology Resource (based in Newcastle and London) and collaborators in the UK and worldwide. So far, we have published our findings on the maternal-fetal interface that sustains pregnancies, the development of blood and immune cells in the fetal liver, skin and kidney and the differentiation of T cells in the thymus.

The data are freely available (https:// developmentcellatlas.ncl.ac.uk). My research group and collaboration network comprise an eclectic and happy mix of researchers with immunology, genomics, computational and software-development expertise.

\section{Muzlifah Haniffa ${ }^{1,2,3}$}

${ }^{1}$ Biosciences Institute, Faculty of Medical Sciences, Newcastle University, Newcastle upon Tyne, UK. ${ }^{2}$ Department of Dermatology and NIHR Newcastle Biomedical Research Centre, Newcastle Hospitals NHS Foundation Trust, Newcastle upon Tyne, UK. ${ }^{3}$ Wellcome Sanger Institute, Wellcome Genome Campus, Cambridge, UK.

e-mail:m.a.haniffa@ncl.ac.uk

Published online: 11 February 2020 https://doi.org/10.1038/s41591-020-0767-X 